\title{
A prospective study of the palpable lesions of the breast and the role of FNAC in these lesions with available histopathology methods
}

\author{
Arzeena Kaleemuddin ${ }^{1}$, K. Suresh ${ }^{2 *}$, C.C Mohan Reddy ${ }^{3}$, Florence Nightingale ${ }^{4}$ \\ ${ }^{1}$ Dr. Arzeena Kaleemuddin, Department of Pathology, SVS Medical College, Mahabubnagar, Telangana, INDIA. ${ }^{2}$ Dr. K. \\ Suresh, Associate Professor, Department of Pathology, Narayana Medical College, Nellore, Andhra Pradesh, INDIA. \\ ${ }^{3}$ Dr. Mohan Reddy, Department of Pathology, SVS Medical College, Mahabubnagar, Telangana, INDIA. ${ }^{4}$ Dr. Florence \\ Nightingale, Department of Pathology, Narayana Medical College, Nellore, Andhra Pradesh, INDIA.
}

Address for Correspondence: Dr. K. Suresh, Associate Professor, Department of Pathology, Narayana Medical College, Nellore, Andhra Pradesh, INDIA. Email: suresh_harithasa@yahoo.com.

\begin{abstract}
Introduction: Benign as well as malignant breast lesions are quite common in Indian population. It is the second most common cancer site after cancer cervix. Fine-needle aspiration cytology (FNAC) is safe, reliable, and time saving outdoor procedure with little discomfort to the patient. Objective: The current study was aimed to study the frequency of various breast lesions on FNAC in a tertiary care center and its histopathological correlation. Methodology: Total of 217 cases of FNAC was conducted in the palpable lesions of the breast. Result: Most of the lesions were noted in the left breast (123) lesions out of which 29 were malignant. The various benign neoplastic lesions seen were 81 patients. Fibroadenoma were found mostly in the age group of 21-30 years. 41 cases of fibrocystic disease found mostly in the age group of 31 to 50 years. The lymph node metastasis was seen in 5 patients with 35 to $55 \mathrm{yrs}$ of age. There were 71 benign neoplastic lesions and 21 cases of malignancy available for histopathology. FNAC was $80.9 \%$ sensitive and $95.89 \%$ specific in diagnosing malignant lesions. Conclusion: A high sensitivity and a high positive predictive value proved that a positive FNAC in the breast means a definite diagnosis of the concerned pathology if compared with the final histology report. Thus, we have no hesitation in concluding that FNAC is a very important preliminary diagnostic test in palpable breast lumps and done by expert hands, the results show a high degree of correlation with the final histopathology report.
\end{abstract}

Keywords: Breast lump, Fine needle aspiration cytology, Histopathology, Malignant lesion

\section{Introduction}

The breast or more specifically the mammary gland is man's insignia of its membership in the class mammalia. It is the upper ventral region of the torso of a primate, in left and right sides. Both men and women develop breasts from the same embryological origin. Due to the higher level of estrogen, breasts become far more prominent than those of men $\&$ are subjected to a constant dynamic role of physiological changes related to pregnancy, lactation, menstruation and menopause. The symptoms of breast disorders, include cyclical mastalgia, lump in the breast, nipple discharge. Benign breast diseases such as mastitis and fibroadenoma of the breast are more common causes of breast disorder symptoms. The first noticeable symptom of breast

Manuscript received: $24^{\text {th }}$ November 2016

Reviewed: $02^{\text {nd }}$ December 2016

Author Corrected: $10^{\text {th }}$ December 2016

Accepted for Publication: $17^{\text {th }}$ December 2016 cancer is typically a lump that feels different from the rest of the breast tissue. More than $80 \%$ of breast cancer cases are discovered when the woman feels a lump.

Worldwide, breast cancer is the most common invasive cancer in women. Breast cancer comprises $22.9 \%$ of invasive cancers in women [1] and $16 \%$ of all female cancers [2]. The incidence of breast cancer varies greatly around the world: it is lowest in less-developed countries and greatest in the more-developed countries. The annual age-standardized incidence rate per 100,000 women in Eastern Asia is around $18 \&$ in South Central Asia are around 22.

The primary risk factors for breast cancer are female sex [3] age, [4] lack of childbearing or breastfeeding [5]. higher hormone levels,[6] race, economic status 


\section{Research Article}

smoking tobacco also increases the risk of breast cancer with the greater the amount of smoking and the earlier in life smoking begins the higher the risk [7].

Thus cancer of the breast remains an elusive disease that has claimed many victims throughout the world from times immemorial. It is both ironic and tragic that a neoplasm arising in an exposed organ readily accessible to examination and clinical diagnosis continues to exert such a heavy toll on human life. Because of cosmetic consideration, fear of loss of feminity and mutilation, there is great hindrance to early diagnosis \& prompt treatment, from times of earliest recorded cases of malignancy.

While screening techniques are useful in determining the possibility of cancer, a further testing is necessary to confirm whether a lump detected on screening is cancer, as opposed to a benign alternative such as a simple cyst.

The noninvasive methods of examination include mammography, Ultrasound and additional tests that are performed in special circumstances such as MR imaging. Both mammography and clinical breast exam, also used for screening, can indicate an approximate likelihood that a lump is cancer, and may also detect some other lesions [8].

The invasive methods include Fine Needle Aspiration Cytology (FNAC). FNAC is a simple technique readily tolerated by the patients, does not require using anesthesia, it involves extraction of a small sample from the lesion for microscopic examination, without interfering with the radiological and gross appearance of the breast. It is quick to perform, inexpensive, needs little equipment and is also preferred in busy surgical outpatient clinics. The report can also be issued in a fairly short duration. Thus this procedure has found increasing acceptance and applicability in clinical use.

Other options for examination include core biopsy, where a section of the breast lump is removed, and eventually all these may require an excision biopsy, where the entire lump is removed or mastectomy is performed if required and a final diagnosis is reached upon.

The present study has been taken up to study the diagnostic value of FNAC in the lesions of the breast, and it has been compared with the available histopathology thus verifying the accuracy of FNAC in detecting the lesions of the breast $\&$ differentiating benign from malignant lesions to aid in the further management of the disease.

\section{Methods}

A study of 217 cases of FNAC was conducted in the palpable lesions of the breast. The study was conducted from august 2009 to july 2011 in SVS medical collegeMahbubnagar. The cases were recorded in a prepared proforma, fine needle aspiration done and cytology studied. All smears were stained by HaematoxylinEosin stain. 94 of these patients underwent surgery and cytological and histopathological results were compared. The results were also compared with other published studies and works by other authors.

The importance of the investigation was explained to the patient. The patient was assured of painlessness of the procedure and consent of the patient was taken. No anaesthesia was used in the present study.

The breast lump was localized accurately $\&$ the patient made comfortable. Skin over the lesion was prepared by cleaning with spirit swab. Lump was fixed with one hand between the thumb and index finger. The needle with a well fitted syringe was inserted into the lesion, perceiving the tissue texture while entering the lesion. Then the piston of the syringe was retracted to create a negative pressure in the needle bore and the syringe while still maintaining the negative pressure was moved back and forth in various directions with the needle still in the lesion. Slowly releasing the negative pressure the needle was retracted from the lesion. The needle was then detached from the syringe, the syringe filled with air and needle replaced. The syringe was held vertical with the tip just above the glass slide and material from the needle expressed onto the glass slide, by pressing the piston of the syringe, thus blowing the contents of the bore of the needle onto the slide.

The procedure was repeated to make another pass into the lesion to get an adequate sample whenever required in some cases. The aspirate was spread evenly and thinly on the glass slide with the help of another slide to make smears. The smears were immediately fixed in a fixative solution $(90 \%$ alcohol) for a minimum period of 20 to 30 minutes and were stained with Haematoxylin and Eosin staining.

After studying the nature of aspirate, the cellularity, the cytomorphology, the alignment of cells, the 


\section{Research Article}

background, the lesions were classified and a diagnosis was given to plan for further treatment and management of the case.

Out of the 217 cases aspirated 94 undergone surgeries. The excised specimens received in the pathology department were examined thoroughly, the gross and cut section findings were noted and several bits were taken from the appropriate sites for processing and paraffin embedding. From each block, sections were cut of 3-5 microns thickness, and stained with Haematoxylin \& Eosin. After studying the histomorphology, the diagnosis was given.

\section{Results}

We included all patients with palpable breast lumps and also few patients with nipple discharge associated with these lumps. Out of these 217 patients 94 patients underwent surgery and histopathological evaluation was done in these cases. There were 10(4.6\%) male patients and 207 (95.4\%) female patients with a male to female sex ratio of around 1:21.

Distribution of the lesions \& Age incidence- Out of total 217 breast aspirations, 23 (10.5\%) were inflammatory \& non neoplastic lesions, $141(64.9 \%)$ were benign \& $44(20.3 \%)$ malignant lesions. There were $7(3.3 \%)$ patients with inadequate smears and $2(0.92 \%)$ patients with smears suspicious for malignancy.

Age of the 217 patients aspirated ranged from 13 years to 76 years with a mean age of $31.5 y r s$.

Most of the cases belonged to the age group of 20 to 40 years, with a maximum number of females affected between 20 to 30 years of age and males affected between 30 to 40 years [Table 1]

Table-1: Age \& Sex wise incidence of breast lesions

\begin{tabular}{|c|c|c|}
\hline & Males & Females \\
\hline Less than 20yrs & 0 & 63 \\
\hline 21-30yrs & 0 & 50 \\
\hline 31-40yrs & 5 & 36 \\
\hline 41-50yrs & 0 & 13 \\
\hline 51-60yrs & 3 & 2 \\
\hline
\end{tabular}

Laterality of the lesions- Most of the lesions were noted in the left breast, with 123 left sided lesions out of which 29 were malignant. There were 76 right sided lesions out of which 14 were malignant and 18 bilateral lesions. Among the bilateral lesions there was only one case of bilateral invasive duct cell carcinoma NOS with associated papillary carcinoma thyroid in a 65 year old female. The other bilateral lesions were all benign neoplastic lesions.

Male patients- There were 10 male patients, out of these 8 patients were diagnosed as gynaecomastia and 2 patients with duct cell carcinoma. Gynaecomastia was bilateral in 5 patients, right sided in 1 \& left sided in 2 cases. Malignancy was left sided in both the patients. One patient was $58 \mathrm{yrs}$ old and the other was $65 \mathrm{yrs}$ old. The histopathological evaluation was available in one patient with gynaecomastia and one patient with duct cell carcinoma. In both these cases a cytological diagnosis was made and proved in histopathology to be the same.

Female patients- There were a total of 207 female patients, out of these $23(11.11 \%)$ were non neoplastic lesions, 135 $(65.21 \%)$ were neoplastic lesions and $42(20.28 \%)$ were malignant. In 7 (3.3\%) cases the FNAC material was not adequate for diagnosis. Females in the age group of 21 to 30 years were mostly affected as far as benign neoplastic conditions were concerned. Malignancies were seen mostly in the age group of 41 to 60 years. 


\section{Research Article}

Breast lesions- The various non-neoplastic lesions seen were 3 cases of simple cysts, 6 cases of acute mastitis mostly in the age group of 21-30years, 10 cases of granulomatous mastitis mostly in the age of 20-40years, 3 cases of galactocoele and one patient with microfilaria. Overall the maximum numbers of cases were seen in the age group of 21-30 years. The various benign neoplastic lesions seen were 81 patients with fibroadenoma seen mostly in the age group of 21-30 years, 41 cases of fibrocystic disease seen mostly in the age group of 31 to 50 years, 3 cases of papilloma in the age group of 41 to 50 years of age, 4 cases of benign phyllodes tumor 2 seen in the later part of the third decade and one each in the $5^{\text {th }} \&$ $6^{\text {th }}$ decades, and lastly 6 cases of nipple discharge diagnosed as negative for malignant cells seen in the $4^{\text {th }}-6^{\text {th }}$ decade [Figure 1]. Among the patients with fibroadenoma 5 cases of giant fibroadenoma were seen with 4 patients below the age of 20 years and 1 patient was 30 years old. All these patients had lesions measuring more than $5 \times 5 \mathrm{cms}$ on examination.[Table 2]
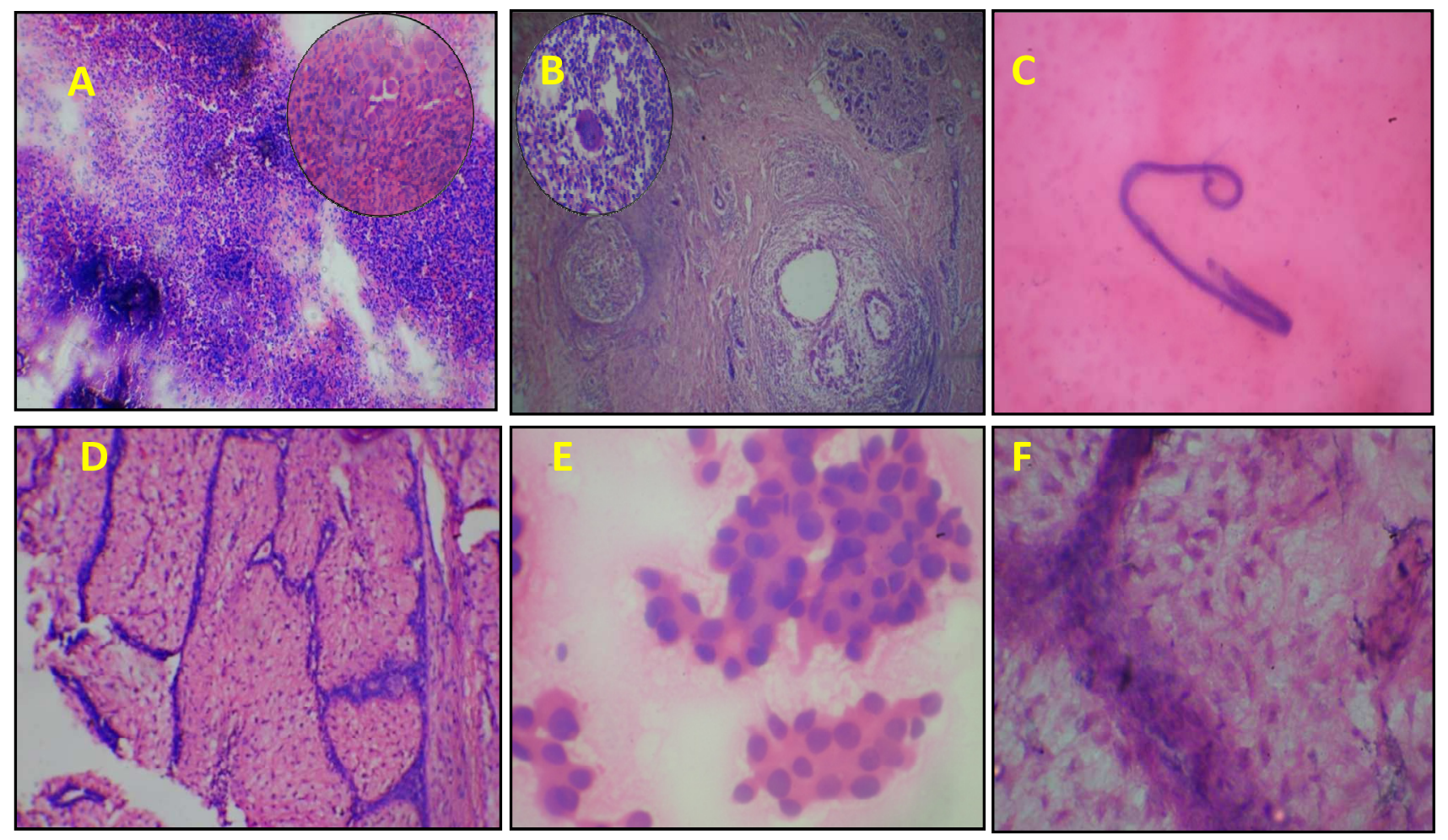

Figure-1: A. Acute mastitis - FNAC 10X, Inset ductal cells admixed at higher magnification 40X. B. Granulomatous mastitis H\&E stained section showing granulomas \& inset showing Giant cell \& chronic inflammatory cell. C. Microfilaria. D. Fibroadenoma - Intraductal pattern H\&E 10X. E. FNAC Fibrocystic disease with apocrine cells H\&E 40 X. F. Benign phyllodes tumor - concentration of cells beneath the epithelium no atypia, no mitotic figures. H\&E $40 \mathrm{X}$.

Table-2: Age wise distribution of the various benign neoplasms

\begin{tabular}{|c|c|c|c|c|c|}
\hline Age groups & Fibroadenoma & $\begin{array}{c}\text { Fibrocystic } \\
\text { disease }\end{array}$ & $\begin{array}{c}\text { Benign phyllodes } \\
\text { tumor }\end{array}$ & Papilloma & Nipple discharge \\
\hline Less than 20yrs & 23 & 0 & 0 & 0 & 0 \\
\hline 21-30yrs & 42 & 4 & 2 & 0 & 0 \\
\hline 31-40yrs & 14 & 18 & 1 & 0 & 2 \\
\hline 41-50yrs & 1 & 13 & 1 & 3 & 1 \\
\hline $\mathbf{5 1 - 6 0 y r s}$ & 1 & 2 & 0 & 0 & 2 \\
\hline 61-70yrs & 0 & 4 & 0 & 0 & 1 \\
\hline
\end{tabular}

There were 42 cases of primary malignant lesions of the breast with 33 cases of duct cell carcinoma mostly in the age group of 51-60 years, 1 case of large cell non-keratinising squamous cell carcinoma of the breast at 55yrs of age with no other focus of a primary squamous cell carcinoma, 1 case of pure mucinous carcinoma at 45 years, 1 case of duct cell carcinoma with mucin production at 45 years $\& 6$ cases of medullary carcinoma breast mostly in the $5^{\text {th }}$ and $6^{\text {th }}$ decade. 


\section{Research Article}

The youngest patient was a 30 year old female with comedo carcinoma and the oldest patient was 72 year old female with invasive duct cell carcinoma NOS [Figure 2].
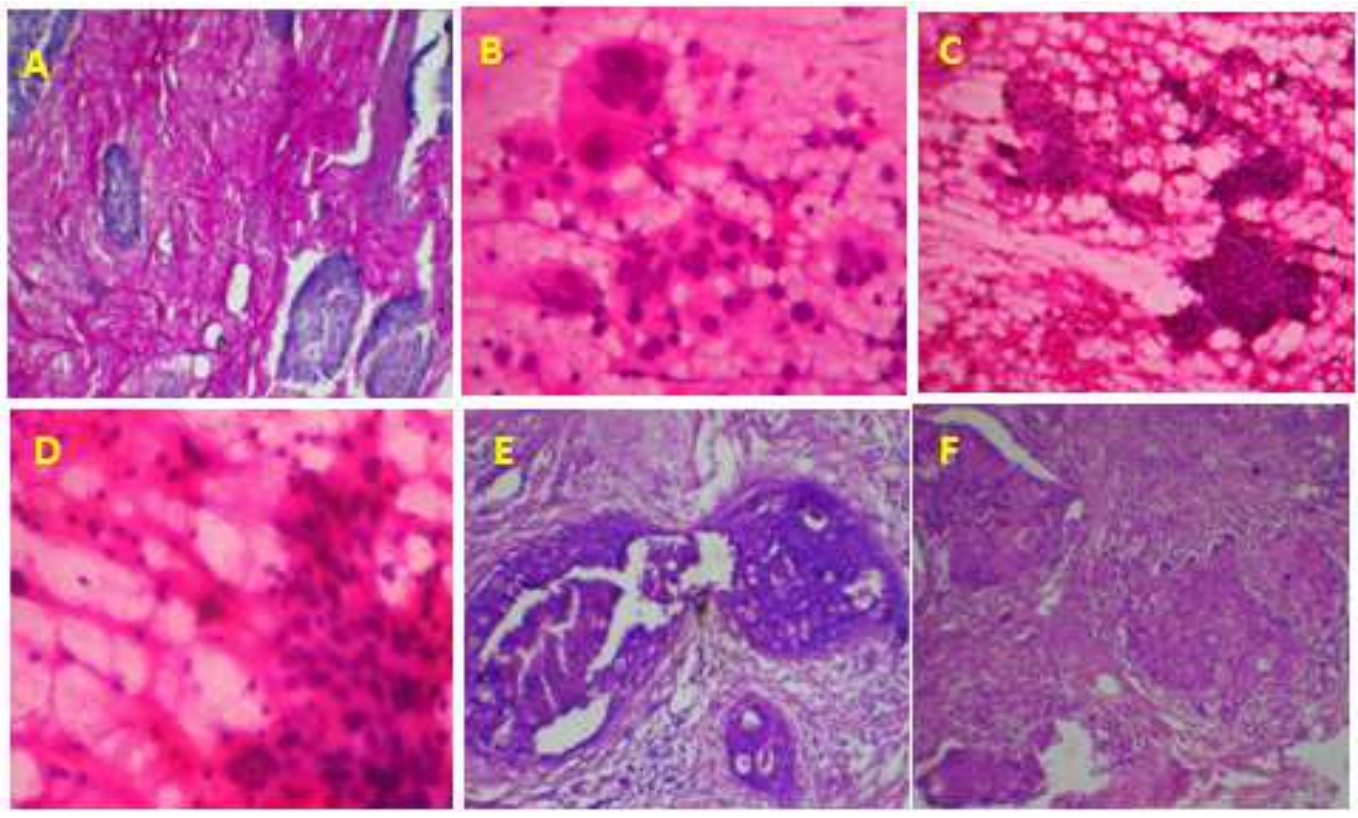

Figure-2: A. Mucinous carcinoma H\&E and mucicarmine stain. B. invasive duct cell carcinoma with tumor giant cells and aggressive looking pleomorphic cells. C. Invasive duct cell carcinoma - secondary deposit against background of lymphotyces. D. smears showing few atypical cells giving a suspicion of malignancy. E. comedo carcinoma. F. Large cell non - keratinizing squamous cell carcinoma.

Metastatic lesions- Among these patients with malignancy, 5 showed metastasis to the axillary group of lymph nodes. The lymph node metastasis was seen in patients in the age group of 35 to $55 \mathrm{yrs}$ of age. It was noted that most of the patients who were diagnosed as having malignancy attained menarche at 13 years of age \& a majority of them had not attained menopause. Most of them had 3 or more children and all gave a positive history of breast feeding.

There were 7 patients with inadequate smears; the repeat FNAC could not be done in 2 cases as the patients had undergone surgery even before repeat FNAC could be suggested.

There was 1 patient where the lump was very small \& repeat FNAC also showed inadequate material thus a conclusive report could not be given. The remaining 4 patients refused repeat FNAC. It was observed that most of these lesions were small with an ill defined breast lump or showed only vague nodularity of the breast.

Out of the 217 patients who came for FNAC, 94 patients underwent surgery and the specimens were fixed in buffered formalin, examined and adequately sampled, the sections were stained with H\&E and the diagnosis was given. In these cases almost all the cytological diagnosis and histopathological diagnosis correlated with each other except for a few cases.

In the non neoplastic/inflammatory lesions 2 cases were available for histopathological evaluation and the cytological \& histopathological diagnosis correlated. These patients were diagnosed as granulomatous mastitis.

Sensitivity \& specificity- There were 71 benign neoplastic lesions and 21 cases of malignancy available for histopathology. There were 3 cases of false positives reported as malignant on FNAC and benign on histopathology.

There were 4 false negatives reported as benign on FNAC \& malignant on histopathology. 17 cases of malignancy were reported in which the cytological diagnosis and histopathological diagnosis correlated. 70 cases of benign neoplasms were reported which were diagnosed as benign on FNAC as well as histopathology. 
Table-3: Sensitivity \& Specificity of FNAC results

\begin{tabular}{|l|c|l|c|}
\hline Sensitivity & $80.9 \%$ & False positive percentage & $4.01 \%$ \\
\hline Specificity & $95.89 \%$ & Likelihood ratio of positive test & 19.69 \\
\hline Positive predictive value & $85 \%$ & Likelihood ratio of negative test & 0.1 \\
\hline Negative predictive value & $94.5 \%$ & Diagnostic accuracy & $92.5 \%$ \\
\hline
\end{tabular}

FNAC has very high sensitivity \& specificity.

\section{Discussion}

A lump in the breast is a common complaint presenting in the surgical out-patient department of all major hospitals, with anxiety regarding a possible malignancy being extremely common. Hence a quick diagnosis of a lump in the breast is essential. Criteria such as cost effectiveness, use of anaesthesia, time between the diagnostic procedure and report, patients' hospital stay and most importantly, reliability in deciding subsequent treatment, are all factors to be taken into account in this regard. Considering patients' comfort, lack of requirement of anaesthesia, rapid analysis and reporting, and an absence of false positive results makes FNAC an ideal initial diagnostic modality in breast lumps. The expansion of FNAC in the primary diagnosis of cancer in the last 30 years has been enormous and hugely successful. Its use in detecting the presence of cancer before surgery and as a guide to rational treatment has been well documented. Countries with most developed aspiration biopsy techniques are Sweden, Slovenia, the USA and India.

The smears were fixed in alcohol and stained with H\&E, most European \& American authors like Barbara et al[9], Arisio et al[10], Ciatto et al[11] etc, have used air dried smears stained with romanowsky stains and PAP stain. We have used wet fixed smears \& H\&E stain only. All pathology specimens underwent a histopathological study, the final report was compared with that of FNAC and the correlation was sought.

In our study the youngest patient with malignancy was a 30 year old female with comedo carcinoma in the left breast. There was also a case of large cell non keratinising squamous cell carcinoma of the breast which was reported as suspicious on FNAC due to the large squamoid appearing cells and inadequate sample which was showing few but atypical cells against a haeomorrhagic background. A case of pure mucinous carcinoma was reported as acute mastitis as there was an associated superficial abscess seen in the patient, and imprint smears only were taken. Most of the patients with malignancy had menarche at the age of 12 to $13 \mathrm{yrs}$, most of the patients had 3 or more number of children and all had breast fed the children. Out of the forty two patients 25 patients had not yet attained menopause while the other 17 had attained menopause.

There were 5 cases with metastasis to the axillary lymph nodes and 1 patient with widely disseminated disease in the form of secondaries in the mandible \& involvement of axillary and cervical group of lymph nodes. Most of these patients were aged between 40 to 50 yrs of age.

In a similar study done by Aditya et al[12] on 50 patients, the age distribution was between fifteen and sixty-five years and the maximum patients were seen in the thirty one to forty year group (30\%). Similar studies done by, Tiwari et al[13] and Ariga et al[14] showed similar age patterns.

In our study, the right breast was involved in 76 patients while the left breast was involved in one hundred \& twenty three patients. Bilateral involvement was seen in eighteen patients. In their series, Aditya et al. showed left breast involvement in 27 patients (54\%) while in the other 23, the right breast was involved [12]. No surgical importance can be attached to this observation since patient selection was in no way dictated by involvement of any particular breast.

12 out of our 217 patients were married. This corresponds to $5.52 \%$ unmarried and $94.5 \%$ married patients in comparison to $74 \%$ of 50 patients who were married in the series by Aditya et al[12]. In both series more married women presented to the out-patient department than unmarried ones. Though not directly related to our study, these figures may indicate the relative reluctance of young unmarried women to present to the out-patients department for a breast examination, 
since benign breast lumps, are definitely not uncommon in the young unmarried patient. Also all those who had children had breastfed their babies.

In our patients, the duration of the lump ranged from 1 week to one and a half years. All the others reported duration in between 2 and 7 months. In comparison, the study by Aditya et al[12] showed the duration of the lumps in a range between 1 month to 18 months with a majority of patients having a history of between 6 months and a year. Earlier presentation in case of our patients was probably a reflection of the increased awareness regarding breast lumps amongst the population.

The commonest pathology found in our patients was fibroadenoma in 81 patients. In their study on 91 patients, Tiwari et al.[13] also reported fibroadenoma as the commonest pathology (49.6\%). Other important conditions such as subareolar abscess, invasive ductal cancer, breast abscess, fibrocystic disease, duct ectasia, and galactocoele ranged from 5.5\% to $7.7 \%$.

Size of the needle used for FNAC has often been a point for discussion since patient comfort and patient friendliness is an important aspect of FNAC as a superior diagnostic procedure. Disadvantages of a finer needle were an inadequate aspirate while disadvantages of a thicker needle included pain and hematoma formation.

All our patients underwent FNAC using a no. 21 needle with no patient discomfort and none of the patients complained of any untoward side effects. Walker et al.[15] compared the use of $21 \mathrm{G}$ and $23 \mathrm{G}$ needles for FNAC in breast lumps; 125 patients were included; 61 and 64 patients underwent FNAC with a $21 \mathrm{G}$ and $23 \mathrm{G}$ needle, respectively. A chi squared test had showed no statistical difference in the results whichever needle was used in their study.

Expertise of the person performing and interpreting the fine-needle aspiration often influences results. Yeoh et al.[16] from Hong Kong reported a high proportion of unsatisfactory samples (48\%) with doctors who performed FNAC occasionally.

In relation to breast pathologies there are obvious advantages (apart from the more general ones) in the form of very few false positives in differentiating between benign and malignant lesions, as well as of being diagnostic and therapeutic in most breast cysts. Recurrence of carcinoma of the breast, too, can often be diagnosed. The conditions which have a risk of a false positive result are papillary lesions, atypical epithelial hyperplasia, regenerating epithelial atypia, and atypia of ductal epithelium in a cyst.

A risk of false negative results exists in low grade malignancies, small or complex proliferative lesions as well as in tumours with central necrosis or a small cell carcinoma. Silverman et al.[17] concluded that for palpable lesions, FNAC, as compared to core biopsy, appears to have more predictive value in confirming the diagnosis of carcinoma and locally recurrent disease.

In purely statistical terms, there were no normal individuals in our study, i.e. women without a breast lump. Hence, the ability of Fine-Needle Aspiration Cytology as a diagnostic test to identify correctly those individuals not having disease (i.e., true negatives) could not be noted since in every patient in our study FNAC would reveal some result. Therefore, specificity of FNAC as a whole could not be calculated.

In our results, we had no true negatives. In the absence of true negatives, the predictive value of the negative test is actually zero, since the numerator becomes zero. As with the calculation of specificity for malignant lesions, we broadened the interpretation of our results by calculating the negative predictive value of the test for malignant lesions. This aspect has already been explained the results.

In our study, there were 17 true positives, 4 false negatives, no true negatives but 70 non malignant cases taken as true negatives and 3 false positives. As shown previously, sensitivity and positive predictive value of FNAC in our study were calculated as $80.95 \%$ and $85 \%$, respectively, while specificity and negative predictive value for malignancy were $95.89 \%$ and $94.5 \%$, respectively. 
Research Article

The value of sensitivity of FNAC in our study was $80.95 \%$, an absolute value of $96 \%$ was obtained by the series by Aditya et al.[12] In a series of 240 patients, kocjan [18] reported the sensitivity of FNAC as $95 \%$ whereas Ishikawa et al.[19] showed a sensitivity of $86.3 \%$ for FNAC, Mansoor et al.[20] depicted a value of $92.1 \%$ for FNAC. Table 4 showing the values obtained in several similar studies.

Table-4: Comparison of Sensitivity \& Specificity with Other Studies

\begin{tabular}{|c|c|c|c|c|c|}
\hline Name of the authors & Study group & Sensitivity & Specificity & PPV & NPV \\
\hline $\begin{array}{c}\text { Kocjan G et al[18] } \\
(2008)\end{array}$ & 240 & $95 \%$ & $93 \%$ & $94 \%$ & $93 \%$ \\
\hline $\begin{array}{c}\text { Ishikawa et al [19] } \\
\text { (2007) }\end{array}$ & 382 & $86.3 \%$ & $98.2 \%$ & $92.6 \%$ & $90 \%$ \\
\hline $\begin{array}{c}\text { Mansoor et al.[20] } \\
\text { (2002) }\end{array}$ & 276 & $92.1 \%$ & $98.6 \%$ & $99.4 \%$ & $82.1 \%$ \\
\hline $\begin{array}{c}\text { Zanconati et al.[21] } \\
\text { (2000) }\end{array}$ & 308 & $87.8 \%$ & $95.3 \%$ & $76.6 \%$ & $97.8 \%$ \\
\hline $\begin{array}{c}\text { Present Study } \\
\text { (2011) }\end{array}$ & $\mathbf{9 4}$ & $\mathbf{8 0 . 9 5 \%}$ & $\mathbf{9 5 . 8 9 \%}$ & $\mathbf{8 5 \%}$ & $\mathbf{9 4 . 5 \%}$ \\
\hline
\end{tabular}

Another value is the Likelihood Ratio of positive test which indicates how more often a positive test result occurs in persons with compared to those without the target condition, Likelihood ratio of a negative test result indicates how less likely a negative test result is in persons with the target condition compared to those without the target condition. A $\mathrm{LR}=1$ indicates no diagnostic value, $\mathrm{LR}+>10$ are usually regarded as a 'strong' positive test result, $\mathrm{LR}-<0.1$ are usually regarded as a strong negative test result.

In our study we got a LR+ 19.69, and a LR- 0.1. Our diagnostic accuracy was 92.5\%. With the procedure of performing FNAC fairly standardized by now in all institutions, the only important variable remains the person doing the actual aspiration. A major obstacle in getting better results is removed when only pathologists with experience were performing the aspiration in various studies and when better cyto-histologic correlation is obtained.

\section{Conclusions}

The overall accuracy was $92.5 \%$, the number of false negatives 4 and the number of false positives 3 . A high sensitivity and a high positive predictive value proved that a positive FNAC in the breast means a definite diagnosis of the concerned pathology when compared with the final histology report.

The high specificity and a high negative predictive value for malignancy illustrated the high accuracy of FNAC in the diagnosis of malignancy in the breast. Very importantly, a report negative for malignancy was highly accurate $(>92 \%)$ in predicting an absence of malignancy.

Thus, we have no hesitation in concluding that FNAC is a very important preliminary diagnostic test in palpable breast lumps, and done by expert hands, the results show a high degree of correlation with the final histopathology report.

Funding: Nil, Conflict of interest: None initiated, Permission from IRB: Yes

\section{References}

1. Gøtzsche PC, Jørgensen KJ: Screening for breast cancer with mammography. Cochrane Database Syst Rev. 2013 Jun 4;(6):CD001877. doi: 10.1002/14651858.CD001877.pub5.

2. Institute NC: SEER stat fact sheets: Breast cancer. 2008.

3. Giordano SH, Cohen DS, Buzdar AU, Perkins G, Hortobagyi GN: Breast carcinoma in men: a population-based study. Cancer. 2004 Jul 1;101(1):517.

4. Al Zahrani NM, Soomro S, Memon AG: Breast Cancer Diagnosis and Treatment of Prophetic Medicine Using Expert System. Journal of Information \& Communication Technology 2010, 4(2):20-26.

5. Cancer CGoHFiB: Breast cancer and breastfeeding: collaborative reanalysis of individual data from 47 epidemiological studies in 30 countries, including 50 302 women with breast cancer and 96973 women 


\section{Research Article}

without the disease. The Lancet 2002, 360(9328):187195.

6. Yager JD, Davidson NE: Estrogen carcinogenesis in breast cancer. New England Journal of Medicine 2006, 354(3):270-82.

7. Xue F, Willett WC, Rosner BA, Hankinson SE, Michels KB: Cigarette smoking and the incidence of breast cancer. Arch Intern Med. 2011 Jan 24;171(2):125-33. doi: 10.1001/archinternmed.2010.503.

8. Organization WH: Breast cancer: prevention and control. World Health Organization[Online] 2012.

9. Florentine BD, Staymates B, Rabadi M, Barstis J, Black A; Cancer Committee of the Henry Mayo Newhall Memorial Hospital. The reliability of fineneedle aspiration biopsy as the initial diagnostic procedure for palpable masses: a 4-year experience of 730 patients from a community hospital-based outpatient aspiration biopsy clinic. Cancer. 2006 Jul 15;107(2):406-16.

10. Arisio R, Cuccorese C, Accinelli G, Mano MP, Bordon R, Fessia L: Role of fine $\square$ needle aspiration biopsy in breast lesions: Analysis of a series of 4,110 cases. Diagnostic cytopathology 1998, 18(6):462-467.

11. Ciatto S, Cecchini S, Grazzini G, Iossa A, Bartoli D, Cariaggi M, Bulgaresi P: Positive predictive value of fine needle aspiration cytology of breast lesions. Acta cytologica 1988, 33(6):894-8.

12. Khemka A, Chakrabarti N, Shah S, Patel V: Palpable breast lumps: Fine-needle aspiration cytology versus histopathology: A correlation of diagnostic accuracy. The internet journal of surgery 2009, 18(1).

13. Tiwari M. Role of fine needle aspiration cytology in diagnosis of breast lumps. Kathmandu Univ Med J (KUMJ). 2007 Apr-Jun;5(2):215-7.
14. Ariga R, Bloom K, Reddy VB, Kluskens L, Francescatti D, Dowlat K, Siziopikou P, Gattuso P: Fine-needle aspiration of clinically suspicious palpable breast masses with histopathologic correlation. The American journal of surgery 2002, 184(5):410-413.

15. Walker S: A randomized controlled trial comparing a $21 \mathrm{G}$ needle with a $23 \mathrm{G}$ needle for fine needle aspiration of breast lumps. Journal of the Royal College of Surgeons of Edinburgh 1998, 43(5):322-323.

16. Yeoh G, Chan K: Fine needle aspiration of breast masses: an analysis of 1533 cases in private practice. Hong Kong Med J. 1998 Sep;4(3):283-288.

17. Silverman JF, Elsheikh TM, Singh HK: The role of fine needle aspiration cytology of the breast in the core biopsy era. Pathology Case Reviews 2007, 12(1):44-48.

18. Kocjan G: Fine needle aspiration cytology: diagnostic principles and dilemmas: Springer Science \& Business Media; 2006.

19. Ishikawa T, Hamaguchi Y, Tanabe M, Momiyama N, Chishima T, Nakatani Y, Nozawa A, Sasaki T, Kitamura H, Shimada H: False-positive and falsenegative cases of fine-needle aspiration cytology for palpable breast lesions. Breast cancer 2007, 14(4):388392.

20. Mansoor I, Jamal AA: Role of fine needle aspiration in diagnosing breast lesions. Saudi medical journal 2002, 23(8):915-20.

21. Zanconati F, Bonifacio D, Falconieri G, Di Bonito L: Role of fine $\square$ needle aspiration cytology in nonpalpable mammary lesions: A comparative cytohistologic study based on 308 cases. Diagnostic cytopathology 2000, 23(2):87-91.

\section{How to cite this article?}

Arzeena Kaleemuddin, K. Suresh, C.C Mohan Reddy, Florence Nightingale. A prospective study of the palpable lesions of the breast and the role of FNAC in these lesions with available histopathology methods. Trop J Path Micro 2016;2(3):192-200.doi: 10.17511/jopm.2016.i03.19 